\title{
Outcome of children with severe traumatic brain injury who are treated with decompressive craniectomy
}

\author{
Maroun J. Mhanna, MD, MPH, ${ }^{1}$ Wael El Mallah, MD, ${ }^{1}$ Margaret Verrees, MD, ${ }^{2}$ Rajiv Shah, $M D,{ }^{3}$ and \\ Dennis M. Super, MD, MPH ${ }^{1}$
}

Departments of ${ }^{1}$ Pediatrics, ${ }^{2}$ Neurosurgery, and ${ }^{3}$ Radiology, MetroHealth Medical Center, Cleveland, Ohio

OBJECT Decompressive craniectomy (DC) for the management of severe traumatic brain injury (TBI) is controversial. The authors sought to determine if DC improves the outcome of children with severe TBI.

METHODS In a retrospective, case-control study, medical records of all patients admitted to the pediatric ICU between May 1998 and May 2008 with severe TBI and treated with DC were identified and matched to patients who were treated medically without DC. Medical records were reviewed for patients' demographic data and baseline characteristics.

RESULTS During the study period, 17 patients with severe TBI treated with DC at a median of 2 hours (interquartile range [IQR] 1-14 hours) after admission were identified and matched to 17 contemporary controls. On admission, there were no differences between $D C$ and control patients regarding age $(10.2 \pm 5.9$ years vs $12.4 \pm 5.4$ years, respectively [mean \pm SD]), sex, weight, Glasgow Coma Scale score (median 5 [IQR 3-7] vs 4 [IQR 3-6], respectively; $p=0.14$ ), or the highest intracranial pressure (median 42 [IQR 22-54] vs 30 [IQR 21-36], respectively; $p=0.77$ ). However, CT findings were significant for a higher rate of herniation and cerebral edema among patients with $D C$ versus controls $(7 / 17$ vs $2 / 17$, respectively, had herniation [ $p=0.05]$ and $14 / 17$ vs $6 / 17$, respectively, had cerebral edema [ $p=0.006]$ ). Overall there were no significant differences in survival between patients with DC and controls $(71 \%$ [12/17] vs $82 \%$ [14/17], respectively; $p=0.34$ ). However, among survivors, at 4 years (IQR $1-6$ years) after the TBI, $42 \%(5 / 12)$ of the DC patients had mild disability or a Glasgow Outcome Scale score of 5 vs none $(0 / 14)$ of the controls $(p=0.012)$.

CONCLUSIONS In this retrospective, small case-control study, the authors have shown that early DC in pediatric patients with severe TBI improves outcome in survivors. Future prospective randomized controlled studies are needed to confirm these findings.

http://thejns.org/doi/abs/10.3171/2014.10.PEDS14117

KEY WORDS decompressive craniectomy; pediatric; traumatic brain injury; outcome; trauma

$\mathrm{H}$ EAD trauma is one of the leading causes of death in children..$^{12}$ Various medical methods have been used to decrease elevated intracranial pressure (ICP), including osmotic diuresis, sedation, and pentobarbital-induced coma. The use of surgical techniques such as decompressive craniectomy (DC) to lower ICP has been proposed in severe traumatic brain injury (TBI)..$^{15,25}$ However, the use of DC remains controversial, ${ }^{8}$ and if the procedure is to be done, a craniectomy that is too small can be detrimental to the patient. ${ }^{26}$ Craniectomy has been shown to improve survival in animal models and in clini- cal settings. 5,16,27 Pediatric patients tend to have a better outcome than adult patients, and can recover from very high ICPs. ${ }^{4}$ In the current study we sought to determine if DC improves survival and/or quality of life in pediatric patients with severe TBI.

\section{Methods}

\section{Patient Population}

Medical records of all pediatric trauma patients with severe TBI who were admitted to our pediatric ICU (PICU)

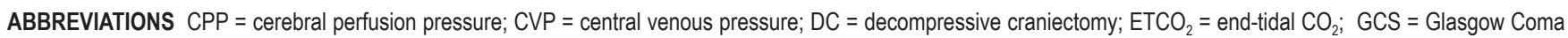
Scale; GLM = general linear model; GOS = Glasgow Outcome Scale; HR = heart rate; ICP = intracranial pressure; IQR = interquartile range; MAP = mean arterial pressure; PICU = pediatric ICU; PRISM = Pediatric Risk of Mortality; TBI = traumatic brain injury.

ACCOMPANYING EDITORIAL See pp 505-507. DOI: 10.3171/2014.11.PEDS14562.

SUBMITTED March 10, 2014. ACCEPTED October 14, 2014.

INCLUDE WHEN CITING Published online July 31, 2015; DOI: 10.3171/2014.10.PEDS14117.

DISCLOSURE The authors report no conflict of interest concerning the materials or methods used in this study or the findings specified in this paper. 
in a Level I trauma center between May 1998 and May 2008 were identified by searching our electronic database. Our inclusion criteria consisted of all pediatric trauma patients $(<18$ years of age) with severe TBI (defined as a Glasgow Coma Scale (GCS) score $\leq 8$ on admission). Our exclusion criteria consisted of all patients who died within the first 24 hours after their TBI and patients who did not have severe TBI (GCS score $>8$ ).

Each patient with severe TBI and who had a DC within the first 48 hours of admission for the management of elevated ICP was matched to a control patient who had severe TBI but did not have a DC for the management of elevated ICP and who was admitted before or after the DC patient (a contemporary match). The decision to perform a DC was at the discretion of the treating neurosurgeon.

All medical records were reviewed for patients' demographic data and baseline characteristics including age, sex, weight, GCS score, Pediatric Risk of Mortality (PRISM) score, cause of injury, and head CT scan findings on admission. All admission CT scans were reviewed by the same neuroradiologist (R.S.) who was blinded to the surgical or medical management of the patients studied. Medical records were also reviewed for physiological variables such as ICP, cerebral perfusion pressure (CPP), mean arterial pressure (MAP), heart rate (HR), and central venous pressure (CVP). Physiological variables were extracted from the daily nursing flow sheets and daily averages were calculated for each patient. Physiological variables were manually entered by the nursing staff at least hourly, and sometimes more frequently depending on the patients' acuity of illness and frequency of critical events.

Interventions that can lower ICP were also reviewed, including the use of CSF drainage, mannitol, hypertonic saline $(3 \%)$, hypocapnea $\left(\mathrm{PaCO}_{2}\right.$ between 30 and $35 \mathrm{~mm}$ $\mathrm{Hg}$ ), barbiturates, and body temperature. Daily average end-tidal $\mathrm{CO}_{2}\left(\mathrm{ETCO}_{2}\right)$, daily morning serum osmolarity, and serum sodium were also reviewed.

Patients' outcomes were reviewed, including survival, Glasgow Outcome Scale (GOS) score, days of mechanical ventilation, days of PICU stay, and length of hospital stay. The need for a tracheostomy or a gastric feeding tube was documented. The number of days of ICP monitoring was also documented.

The study was approved by our institutional review board at MetroHealth Medical Center.

\section{Statistical Analysis}

For nominal data the chi-square and Fisher exact tests were used, and for continuous data paired and unpaired t-tests were used when appropriate. Values were expressed as the mean $\pm \mathrm{SD}$ or as median and interquartile range (IQR). A p value $\leq 0.05$ was considered statistically significant. The general linear model (GLM) with repeated measures was used for comparison between the DC and the control group of daily ICP, CPP, temperature, MAP, $\mathrm{CVP}, \mathrm{ETCO}_{2}$, serum osmolarity, and serum sodium.

\section{Results}

During the study period 249 patients with severe TBI were admitted to our PICU; 17 patients with severe TBI
TABLE 1. Demographic data and baseline characteristics in 34 patients with severe TBI

\begin{tabular}{|c|c|c|c|}
\hline Characteristic & DC & Control & $\begin{array}{c}p \\
\text { Value }\end{array}$ \\
\hline No. of patients & 17 & 17 & \\
\hline Mean age in yrs, $\pm S D$ & $10.2 \pm 5.9$ & $12.4 \pm 5.4$ & 0.28 \\
\hline Sex (\% male) & $9(53 \%)$ & $11(65 \%)$ & 0.48 \\
\hline Mean weight in $\mathrm{kg}, \pm \mathrm{SD}$ & $45.4 \pm 28.1$ & $53.9 \pm 29.3$ & 0.40 \\
\hline $\begin{array}{l}\text { Median GCS score on } \\
\text { admission }\end{array}$ & 5 (IQR 3-7) & $4($ IQR 3-6) & 0.14 \\
\hline Median PRISM score & 13 (IQR 9-21) & 13 (IQR 9-17) & 0.68 \\
\hline \multicolumn{4}{|l|}{ Cause of injury } \\
\hline MVA & $10(59 \%)$ & $13(76 \%)$ & 0.23 \\
\hline ATV & $2(12 \%)$ & $2(12 \%)$ & 1.00 \\
\hline Fall & $2(12 \%)$ & $1(6 \%)$ & 0.50 \\
\hline Others & $3(18 \%)$ & $1(6 \%)$ & 0.30 \\
\hline \multicolumn{4}{|l|}{ CT findings } \\
\hline $\begin{array}{l}\text { Extradural hemor- } \\
\text { rhage }\end{array}$ & $13(76 \%)$ & $7(41 \%)$ & 0.03 \\
\hline $\begin{array}{l}\text { Intracerebral hemor- } \\
\text { rhage }\end{array}$ & $14(82 \%)$ & $12(71 \%)$ & 0.34 \\
\hline Cerebral herniation & $7(41 \%)$ & $2(12 \%)$ & 0.05 \\
\hline Midline shift & $8(47 \%)$ & $3(18 \%)$ & 0.07 \\
\hline Skull fracture & $11(65 \%)$ & $4(23 \%)$ & 0.01 \\
\hline Cerebral edema & $14(82 \%)$ & $6(35 \%)$ & 0.006 \\
\hline Effaced cisterns & $10(59 \%)$ & $6(35 \%)$ & 0.16 \\
\hline
\end{tabular}

ATV = all-terrain vehicle; $M V A=$ motor vehicle accident.

and DC were identified, and they were matched with 17 contemporary controls. Patients' demographic data and baseline characteristics are summarized in Table 1. There were no differences between the 2 groups regarding patients' age, sex, weight, GCS score on admission, PRISM score, or cause of their TBI. However, in comparison with their controls, patients who had a DC had a higher percentage of extradural hemorrhage, skull fractures, cerebral herniations, and cerebral edema underlying a more severe TBI on their admitting CT scans. All patients who were treated with DC had their surgical intervention at a median of 2 hours (IQR 1-14 hours) after their admission.

The different physiological parameters and cointerventions used for the management of ICP during the first 24 hours following the TBI are summarized in Table 2. There were no differences between the 2 groups regarding their average ICP, CPP, MAP, HR, or CVP. There were also no differences in the percentage use of mannitol, hypertonic saline, hypocapnea, and barbiturate-induced coma or body temperature. There were no differences in daily fluid intake between the 2 groups. However, the group of patients who had a DC had fewer CSF drainage devices in place in comparison with the control group.

The highest ICP on admission was comparable between the DC and control group (median 42 [IQR 22-54] $\mathrm{mm} \mathrm{Hg}$ vs 30 [IQR 21-36] mm Hg, respectively, $\mathrm{p}=0.77$ ). The highest ICP on the second day of admission was also comparable between the DC and control group (median 35 [IQR 27-46] $\mathrm{mm} \mathrm{Hg}$ vs 33 [IQR 25-47] $\mathrm{mm} \mathrm{Hg}$, re- 
TABLE 2. Physiological variables and cointerventions used for the management of ICP during the first 24 hours after TBI*

\begin{tabular}{|c|c|c|c|}
\hline Variable & DC & Control & $\begin{array}{c}p \\
\text { Value }\end{array}$ \\
\hline No. of patients & 17 & 17 & \\
\hline $\mathrm{ICP}$ in $\mathrm{mm} \mathrm{Hg}$ & $30(21-42)$ & $27(19-33)$ & 0.99 \\
\hline $\mathrm{CPP}$ in $\mathrm{mm} \mathrm{Hg}$ & $62(49-67)$ & $61(57-67)$ & 0.64 \\
\hline MAP in $\mathrm{mm} \mathrm{Hg}$ & $80(64-93)$ & $76(68-97)$ & 0.95 \\
\hline HR in bpm & $109(83-128)$ & $114(104-153)$ & 0.21 \\
\hline CVP in $\mathrm{mm} \mathrm{Hg}$ & $7(6-8)$ & $8(5-9)$ & 0.83 \\
\hline CSF drainage & $11(65 \%)$ & $17(100 \%)$ & 0.01 \\
\hline Mannitol & $3(18 \%)$ & $4(23 \%)$ & 0.50 \\
\hline Hypertonic saline & $8(47 \%)$ & $7(41 \%)$ & 0.72 \\
\hline $\mathrm{PaCO}_{2} \dagger$ & $8(47 \%)$ & $8(47 \%)$ & 1.00 \\
\hline Barbiturates & $5(29 \%)$ & $4(23 \%)$ & 0.50 \\
\hline $\begin{array}{l}\text { Mean fluid intake in ml/ } \\
\text { kg/day, } \pm \text { SD }\end{array}$ & $125 \pm 91$ & $115 \pm 66$ & 0.71 \\
\hline Temperature in ${ }^{\circ} \mathrm{C}$ & $36(36-38)$ & $38(36-39)$ & 0.16 \\
\hline
\end{tabular}

spectively, $\mathrm{p}=0.52$ ). There was a decrease in the highest ICP between the first day and the second day following DC (the highest ICP decreased from 42 [IQR 22-54] mm $\mathrm{Hg}$ on Day 1 to 35 [IQR 27-46] mm Hg on Day 2, p = 0.18 ); however, the difference did not reach a statistical significance.

During the first 5 days following the TBI there were no differences in daily average ICP (GLM, $p=0.67$ ) or CPP (GLM, $p=0.98$ [Fig. 1]) between the 2 groups. There were also no differences between the DC and control groups regarding daily body temperatures (GLM, $\mathrm{p}=0.11$ ), MAP (GLM, $p=0.50$ ), or CVP (GLM, $p=0.28$ [Fig. 2]). There were also no differences between the DC and control groups regarding daily $\mathrm{ETCO}_{2}(\mathrm{GLM}, \mathrm{p}=0.53)$, serum osmolarities (GLM, $\mathrm{p}=0.61$ ), or serum sodium (GLM, $\mathrm{p}$ $=0.55$ [Fig. 3]).

There were no differences in survival or GOS scores between the 2 groups. However, among survivors, all patients who had a GOS score of 5 (or mild disability) at follow-up had a DC; and none of the control patients had a GOS score of 5 at follow-up. Among the 5 patients who had a DC and GOS score of 5, only 1 patient had an extradural hematoma, along with multiple other contusions and intracranial hemorrhages.

There were no differences in the rate of tracheostomies, gastric tubes, days of ICP monitoring, days of ventilation, PICU length of stay, or hospital length of stay between the 2 groups (Table 3). Our patients were followed for a median of 4 years (IQR 1-6 years).

Among the 17 patients who had a DC, 6 patients underwent the surgery (DC) for a hematoma evacuation, and in 11 patients it was performed for increased ICP only. All DCs were unilateral except in 1 case, in which the DC was bifrontal; and all DCs were performed with dural opening. Also, all DCs were equally distributed among the treating
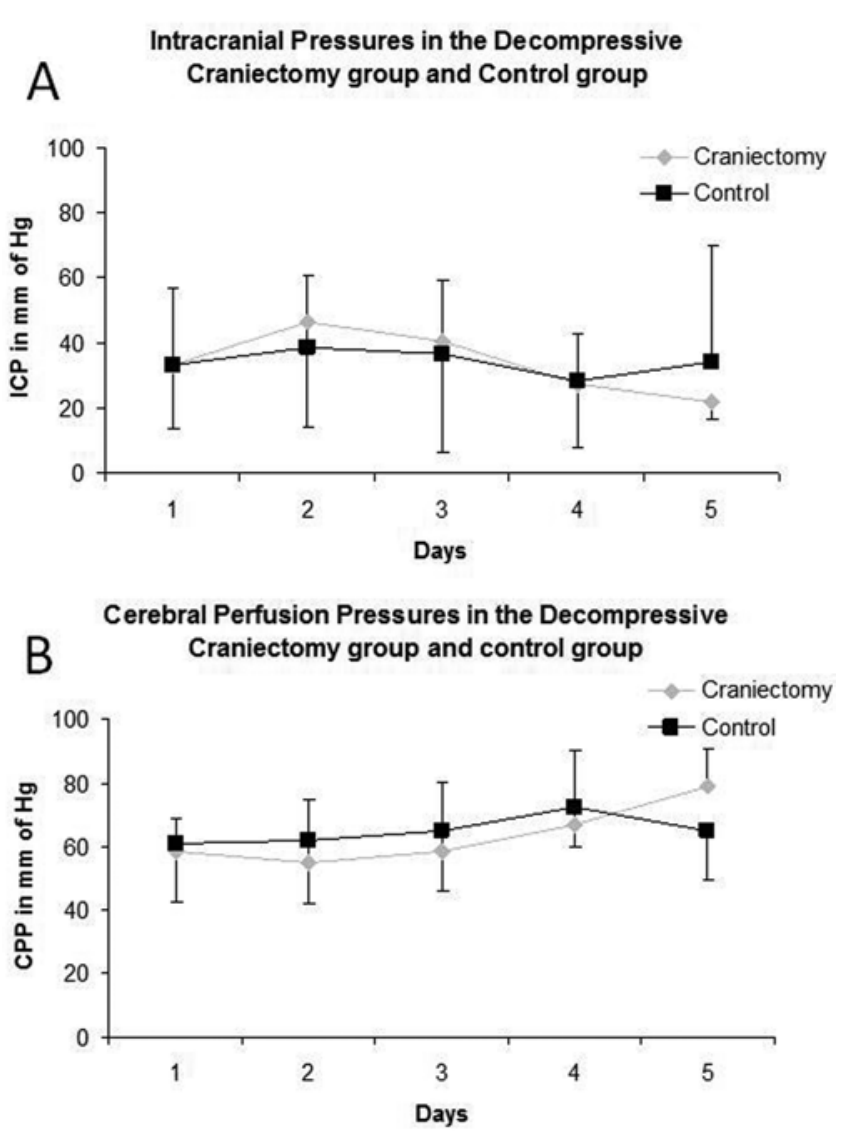

FIG. 1. Graphs showing daily average ICPs (A) and CPPs (B) in the DC and control groups. There were no statistically significant differences between the groups (GLM analysis: $p=0.67$ for ICP, $p=0.98$ for CPP).

physicians-during the study period, there were four neurosurgeons involved in the management of patients with TBI; two of the surgeons performed 5 DCs each, one performed 4, and one performed 3.

Among the 9 patients who presented with cerebral herniation on their brain CT scan, 7 underwent a DC and 2 did not. Among patients who presented with cerebral herniation and had a DC, 57\% (4/7) survived. Of these, 2 survived with a GOS score of 3 (severe disability), 1 survived with a GOS score of 4 (moderate disability), and 1 survived with a GOS score of 5 (mild disability). Neither of the 2 patients who presented with cerebral herniation and did not have a DC survived.

Overall there was a significant difference in outcome between the DC group and the control group regarding the number of patients who died or had any disability following their TBI (71\% [12/17] vs $100 \%$ [17/17], respectively; $\mathrm{p}=0.022$ ).

\section{Discussion}

We have shown in a retrospective, case-control study, that DC in pediatric patients with severe TBI improves morbidity without improvement in mortality rates. A DC within the first hours of admission improves the neurological outcome in survivors of pediatric TBI. 

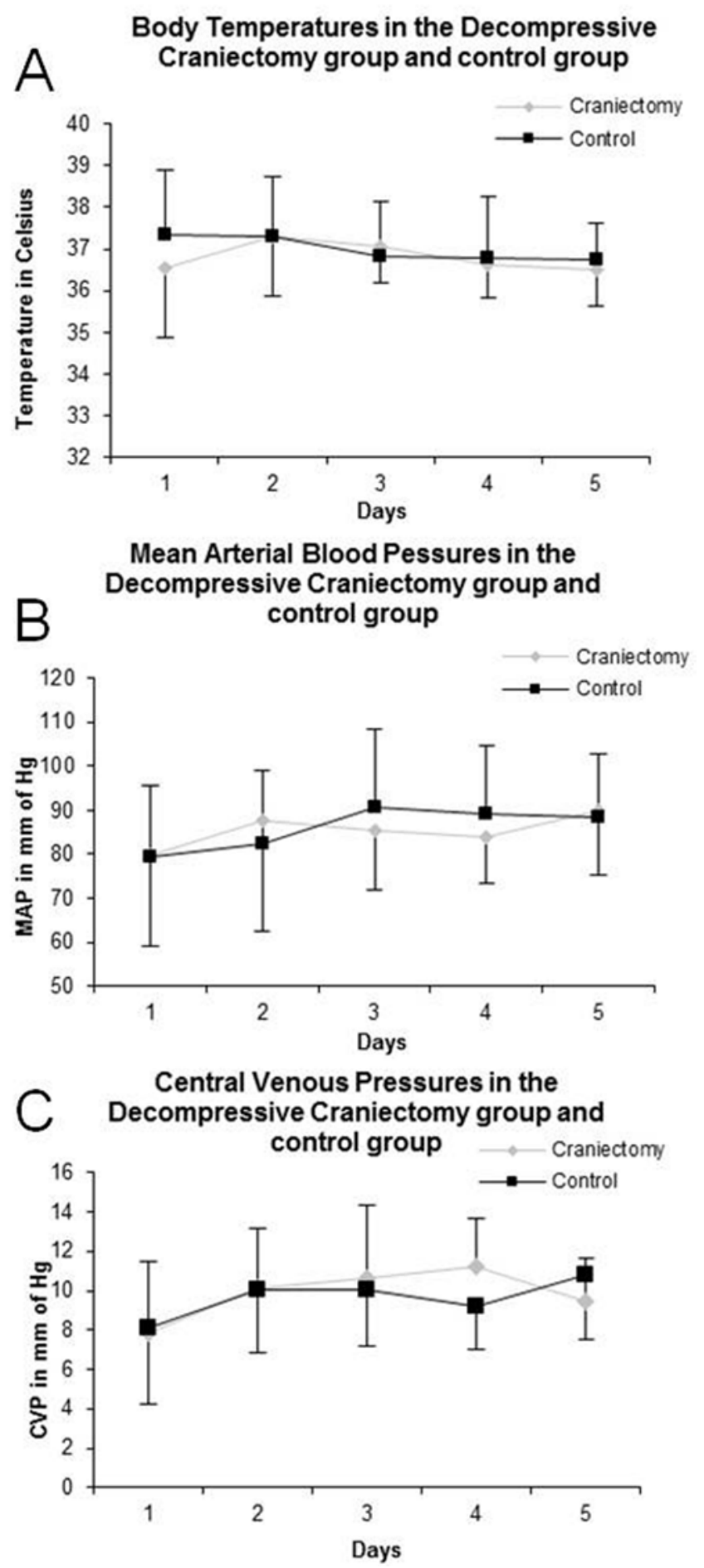

FIG. 2. Graphs showing daily temperatures (A), MAPs (B), and CVPs (C) in the DC and control groups. There were no statistically significant differences between the groups (GLM analysis: $p=0.11$ for daily temperatures, $p=0.50$ for MAPs, and $p=0.28$ for CVPs).

Several studies have shown a favorable outcome after DC in children with severe TBI..$^{6,711,18-21,23}$ However, others have shown a poor outcome after DC in children with severe TBI, when DC was done for increased ICP only, ${ }^{13}$ and in adults with severe TBI and refractory raised ICP. ${ }^{3}$
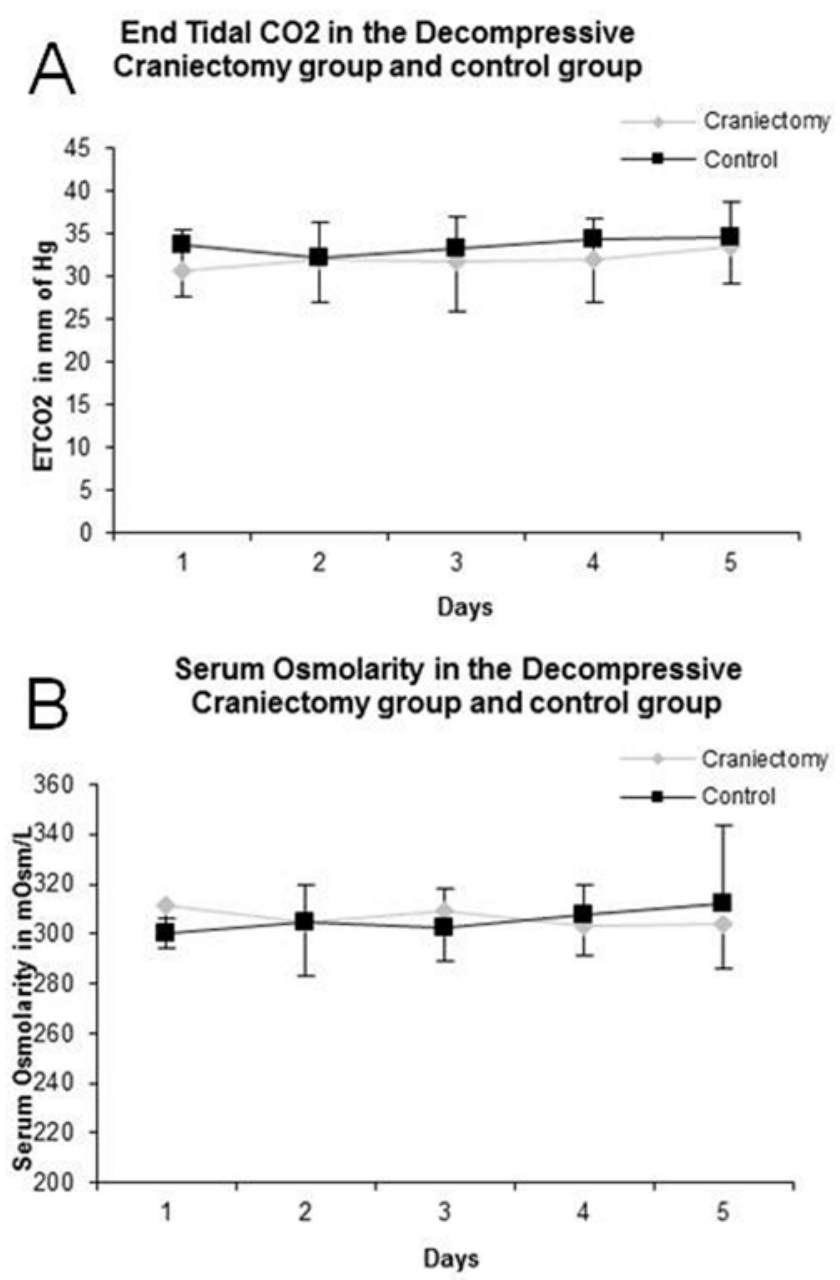

Serum Sodium in the Decompressive Craniectomy group and control group

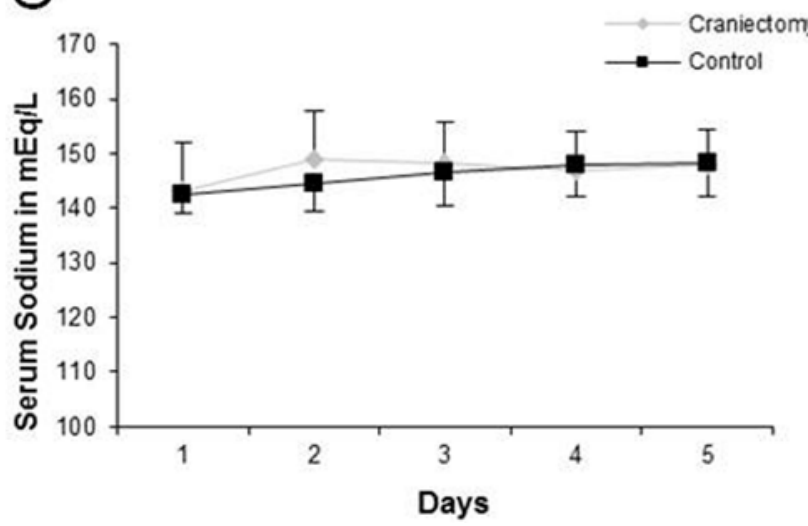

FIG. 3. Graphs showing daily average $\mathrm{ETCO}_{2}$ (A), daily morning serum osmolarities (B), and morning serum sodium (C) in the DC and control groups. There were no statistically significant differences between the groups (GLM analysis: $p=0.53$ for $\mathrm{ETCO}_{2}, p=0.61$ for serum osmolarities, and $p=0.55$ for serum sodium).

Polin et al., in a case-control study of adults and pediatric patients, have shown a favorable outcome in patients who underwent craniotomies, with a mortality rate of $23 \%(8 / 35),{ }^{18}$ which is similar to our mortality rate of 
TABLE 3. Outcome in 34 patients with severe TBI*

\begin{tabular}{|c|c|c|c|}
\hline Outcome & $\mathrm{DC}$ & Control & $\begin{array}{c}\mathrm{p} \\
\text { Value }\end{array}$ \\
\hline No. of patients & 17 & 17 & \\
\hline Survival & $12 / 17(71 \%)$ & $14 / 17(82 \%)$ & 0.34 \\
\hline Median GOS score $†$ & 4 (IQR 3-5) & 3 (IQR 3-4) & 0.09 \\
\hline GOS Score $5 \dagger$ & $5 / 12(42 \%)$ & $0 / 14(0 \%)$ & 0.012 \\
\hline Days of ICP monitoring & $4.2 \pm 2.8$ & $7.6 \pm 7.5$ & 0.10 \\
\hline Tracheotomy† & $4 / 12(33 \%)$ & $2 / 14(14 \%)$ & 0.24 \\
\hline Days of ventilation $\dagger$ & $7.6 \pm 6.4$ & $10.1 \pm 7.4$ & 0.38 \\
\hline Gastric tube $\dagger$ & $5 / 12(42 \%)$ & $6 / 14(43 \%)$ & 0.63 \\
\hline $\begin{array}{l}\text { PICU length of stay in } \\
\text { days } \dagger\end{array}$ & $18.1 \pm 14.9$ & $15.3 \pm 9.1$ & 0.57 \\
\hline $\begin{array}{l}\text { Hospital length of stay } \\
\text { in days } \dagger\end{array}$ & $23.1 \pm 17.6$ & $21.1 \pm 12.0$ & 0.74 \\
\hline
\end{tabular}

$29 \%(5 / 17)$. In their study, pediatric patients $(n=18)$ had a higher rate of favorable outcome than did adult patients who underwent a DC. Ruf et al., in a case series of 6 children with severe TBI treated with a DC, reported that 3 children were without disability at discharge. ${ }^{19}$ Rutigliano et al., in another series of 6 patients with TBI and treated with DC, found that 5 patients left the hospital either independently or with some form of minimal assistance. ${ }^{20}$

Taylor et al., in a randomized trial of very early DC in children with TBI and sustained intracranial hypertension, reported that $54 \%(7 / 13)$ of their patients who underwent a DC survived with a favorable outcome, in comparison with $14 \%(2 / 14)$ in the control group. ${ }^{23}$ Their findings are similar to ours; $42 \%$ of our patients who had a DC and survived had a favorable outcome, in comparison with none of the patients who were in the control group. The similarity between the 2 studies could be related to the timing of the surgical intervention. In Taylor et al.'s study, the DC was performed at a median of 19 hours (range 7-29 hours) from the time of the injury, whereas in our study the DC was performed at a median of 2 hours (IQR 1-14 hours) from the time of the injury. The similarity in the results could be related to the similarity in the early surgical intervention prior to the development of a secondary, irreversible brain injury following the initial TBI insult.

Josan and Sgouros, also in a retrospective case-control study of 12 patients, reported that early DC (within the first 24 hours of injury) resulted in the survival and favorable outcome (GOS scores of 4 and 5) of all 6 patients who underwent an early DC, versus 66\% (4/6) of their controls who were managed with a nonoperative treatment. ${ }^{11}$ Figaji et al., in a case series of 5 patients with severe TBI, reported that early DC resulted in the survival of all of their patients with a favorable outcome.? In our study, DC did not make a difference in survival, but improved the neurological outcome of the individuals who survived.

In a review by Kan et al. of 51 children with severe TBI who had a DC, 16 children (31.4\%) died, including 5 of 6 children who underwent DC for raised ICP only. ${ }^{13}$ Their findings are similar to ours, whereas our overall mortality rate was $29 \%(5 / 17)$ among patients who had a DC; however, in patients who had a DC for increased ICP only, $27 \%(3 / 11)$ died in our series.

Few pediatric studies have reported outcomes similar to ours following DC for the treatment of TBI. In a study of 23 patients younger than 19 years of age, Jagannathan et al. reported that children with TBI who were treated with DC had a favorable outcome, with a mean GOS score of 4.2 at follow-up..$^{10}$ In a study of 14 children with severe TBI, Pérez Suárez et al. also reported a favorable outcome, with a mean GOS score of 4.4 (range 4-5) at the 2-year follow-up. ${ }^{17}$ In our study the majority of our patients who were treated with DC had a favorable GOS score.

Overall we did not find an improvement in the mortality rate between the DC and control groups. However, we found a significant improvement in outcome in survivors despite major differences in severity of the TBI between the 2 groups. In comparison with the control group, the DC group had a higher percentage of cerebral herniation and cerebral edema at presentation. However, the outcome of the patients who had a DC was better than the medically managed control patients with less severe TBI. Some researchers have shown similar results to our findings. Soukiasian et al. found a similar survival rate between patients treated with a DC or craniotomy; however, their patients who underwent a DC had more collapsed basilar cisterns on CT scans than did patients who had a craniotomy, underscoring the role of DC in severe TBI with preoperative basilar cistern collapse. ${ }^{22}$ Thomale et al., in a follow-up study of children treated with DC for TBI, have also reported similar results to ours. They found that children with TBI who were treated with DC had a lower GCS score initially than the nonsurgically treated patients, but at follow-up the 2 groups had a comparable outcome. ${ }^{24}$

Bor-Seng-Shu et al. have shown that DC results in a significant improvement in cerebral blood flow in most patients with TBI and transtentorial herniation, in a study of 19 patients with TBI and cerebral herniation who underwent a DC. ${ }^{2}$ Heppner et al. have also shown an improvement in cerebral microvascular blood flow following a DC in a study of 6 patients who underwent a DC for TBI. ${ }^{9} \mathrm{In}$ our study, in patients who had a DC, the highest ICP on the first day was elevated, and dropped from a median of 42 $\mathrm{mm} \mathrm{Hg}$ to $35 \mathrm{~mm} \mathrm{Hg}$ the next day following the DC; however, the difference was not statistically significant, most probably because our study was underpowered to show a significant difference. We also did not find a difference between the DC and control patients in daily ICP or CPP during the 5 days following their injuries. However, our DC patients had a more severe TBI on their admission CT scan in comparison with their controls, underlining the importance of DC in improving the cerebral perfusion dynamics in severe TBI, resulting in ICP and CPP values that were similar to those in medically managed patients with less severe TBI.

Hyperosmolar therapy (including mannitol and 3\% saline), CSF drainage, mild hyperventilation, hypothermia, and barbiturates have been recommended for the management of intracranial hypertension in pediatric TBI. ${ }^{1,14} \mathrm{In}$ 
our study we found no differences between the DC and control group regarding the medical management of their ICP; however, the control group had a higher percentage of CSF drainage in comparison with the DC group, an intervention that could have lowered the ICP and improved the CPP in the control group. The decision to use a ventricular CSF drainage device more frequently in the control group and a surgical decompression in the DC group could have been related to the severity of illness and differences in ICP between the 2 groups. It is difficult to determine the rationale for using one intervention over the other by retrospectively reviewing the medical records.

Our study has several limitations; it is a small retrospective case-control study of patients treated over a span of 10 years. To counteract any potential confounders related to a change in the approach to managing TBI over the years, a contemporary control group was used for comparison. Each case was matched to a control patient who was admitted prior to or following that DC case. Another limitation of our study is that it was the decision of the treating neurosurgeon to perform a DC, and that decision could have been biased by the admission CT scan findings. The treating team most probably opted to perform a DC because of herniation, edema, and extraaxial hemorrhage. At our institution, impending herniation, uncontrolled ICP, and a midline shift are factors that typically play a major role in the decision making to perform a DC. Future randomized controlled studies are needed to counteract such bias.

Another limitation of our study is related to the retrospective nature of our data collection. We relied on the nurses' frequency of documentation to extract our data. It is possible that many events could have been missed if they were not recorded by the nursing staff. It is also possible that we might have diluted our numbers by averaging out our daily parameters for statistical analysis.

\section{Conclusions}

Overall we have shown that early DC in pediatric patients with severe TBI does not improve mortality rates, but it does improve outcome in survivors in a retrospective case-control study. Future prospective randomized controlled studies are needed to confirm our findings.

\section{References}

1. Adelson PD, Bratton SL, Carney NA, Chesnut RM, du Coudray HE, Goldstein B, et al: Guidelines for the acute medical management of severe traumatic brain injury in infants, children, and adolescents. Chapter 17. Critical pathway for the treatment of established intracranial hypertension in pediatric traumatic brain injury. Pediatr Crit Care Med 4 (3 Suppl):S65-S67, 2003

2. Bor-Seng-Shu E, Hirsch R, Teixeira MJ, De Andrade AF, Marino R Jr: Cerebral hemodynamic changes gauged by transcranial Doppler ultrasonography in patients with posttraumatic brain swelling treated by surgical decompression. J Neurosurg 104:93-100, 2006

3. Cooper DJ, Rosenfeld JV, Murray L, Arabi YM, Davies AR, D'Urso P, et al: Decompressive craniectomy in diffuse traumatic brain injury. N Engl J Med 364:1493-1502, 2011

4. Dam Hieu P, Sizun J, Person H, Besson G: The place of decompressive surgery in the treatment of uncontrollable post- traumatic intracranial hypertension in children. Childs Nerv Syst 12:270-275, 1996

5. Doerfler A, Forsting M, Reith W, Staff C, Heiland S, Schäbitz WR, et al: Decompressive craniectomy in a rat model of "malignant" cerebral hemispheric stroke: experimental support for an aggressive therapeutic approach. J Neurosurg 85:853-859, 1996

6. Figaji AA, Fieggen AG, Argent A, Peter JC: Surgical treatment for "brain compartment syndrome" in children with severe head injury. S Afr Med J 96:969-975, 2006

7. Figaji AA, Fieggen AG, Peter JC: Early decompressive craniotomy in children with severe traumatic brain injury. Childs Nerv Syst 19:666-673, 2003

8. Hatashita S, Hoff JT: The effect of craniectomy on the biomechanics of normal brain. J Neurosurg 67:573-578, 1987

9. Heppner P, Ellegala DB, Durieux M, Jane JA Sr, Lindner JR: Contrast ultrasonographic assessment of cerebral perfusion in patients undergoing decompressive craniectomy for traumatic brain injury. J Neurosurg 104:738-745, 2006

10. Jagannathan J, Okonkwo DO, Dumont AS, Ahmed H, Bahari A, Prevedello DM, et al: Outcome following decompressive craniectomy in children with severe traumatic brain injury: a 10 -year single-center experience with long-term follow up. J Neurosurg 106 (4 Suppl):268-275, 2007

11. Josan VA, Sgouros S: Early decompressive craniectomy may be effective in the treatment of refractory intracranial hypertension after traumatic brain injury. Childs Nerv Syst 22:1268-1274, 2006

12. Juul N, Morris GF, Marshall SB, Marshall LF: Intracranial hypertension and cerebral perfusion pressure: influence on neurological deterioration and outcome in severe head injury. J Neurosurg 92:1-6, 2000

13. Kan P, Amini A, Hansen K, White GL Jr, Brockmeyer DL, Walker ML, et al: Outcomes after decompressive craniectomy for severe traumatic brain injury in children. J Neurosurg 105 (5 Suppl):337-342, 2006

14. Kochanek PM, Carney N, Adelson PD, Ashwal S, Bell MJ, Bratton S, et al: Guidelines for the acute medical management of severe traumatic brain injury in infants, children, and adolescents - second edition. Pediatr Crit Care Med 13 (Suppl 1):S1-S82, 2012

15. Litofsky NS, Chin LS, Tang G, Baker S, Giannotta SL, Apuzzo ML: The use of lobectomy in the management of severe closed-head trauma. Neurosurgery 34:628-633, 1994

16. Münch E, Horn P, Schürer L, Piepgras A, Paul T, Schmiedek P: Management of severe traumatic brain injury by decompressive craniectomy. Neurosurgery 47:315-323, 2000

17. Pérez Suárez E, Serrano González A, Pérez Díaz C, García Salido A, Martínez de Azagra Garde A, Casado Flores J: Decompressive craniectomy in 14 children with severe head injury: clinical results with long-term follow-up and review of the literature. J Trauma 71:133-140, 2011

18. Polin RS, Shaffrey ME, Bogaev CA, Tisdale N, Germanson T, Bocchicchio B, et al: Decompressive bifrontal craniectomy in the treatment of severe refractory posttraumatic cerebral edema. Neurosurgery 41:84-94, 1997

19. Ruf B, Heckmann M, Schroth I, Hügens-Penzel M, Reiss I, Borkhardt A, et al: Early decompressive craniectomy and duraplasty for refractory intracranial hypertension in children: results of a pilot study. Crit Care 7:R133-R138, 2003

20. Rutigliano D, Egnor MR, Priebe CJ, McCormack JE, Strong N, Scriven RJ, et al: Decompressive craniectomy in pediatric patients with traumatic brain injury with intractable elevated intracranial pressure. J Pediatr Surg 41:83-87, 2006

21. Sahuquillo J, Arikan F: Decompressive craniectomy for the treatment of refractory high intracranial pressure in traumatic brain injury. Cochrane Database Syst Rev 1:CD003983, 2006

22. Soukiasian HJ, Hui T, Avital I, Eby J, Thompson R, Kleisli 
$\mathrm{T}$, et al: Decompressive craniectomy in trauma patients with severe brain injury. Am Surg 68:1066-1071, 2002

23. Taylor A, Butt W, Rosenfeld J, Shann F, Ditchfield M, Lewis E, et al: A randomized trial of very early decompressive craniectomy in children with traumatic brain injury and sustained intracranial hypertension. Childs Nerv Syst 17:154162,2001

24. Thomale UW, Graetz D, Vajkoczy P, Sarrafzadeh AS: Severe traumatic brain injury in children - a single center experience regarding therapy and long-term outcome. Childs Nerv Syst 26:1563-1573, 2010

25. Venes JL, Collins WF: Bifrontal decompressive craniectomy in the management of head trauma. J Neurosurg 42:429433, 1975

26. Wagner S, Schnippering H, Aschoff A, Koziol JA, Schwab $\mathrm{S}$, Steiner T: Suboptimum hemicraniectomy as a cause of additional cerebral lesions in patients with malignant infarction of the middle cerebral artery. J Neurosurg 94:693-696, 2001
27. Whitfield PC, Patel H, Hutchinson PJ, Czosnyka M, Parry D, Menon D, et al: Bifrontal decompressive craniectomy in the management of posttraumatic intracranial hypertension. Br J Neurosurg 15:500-507, 2001

\section{Author Contributions}

Conception and design: Mhanna, Verrees. Acquisition of data: Mhanna, El Mallah, Verrees, Shah. Analysis and interpretation of data: Mhanna, Super. Drafting the article: Mhanna. Critically revising the article: Mhanna, Super. Reviewed submitted version of manuscript: Mhanna, Verrees. Approved the final version of the manuscript on behalf of all authors: Mhanna. Statistical analysis: Mhanna, Super. Read CT scans: Shah.

\section{Correspondence}

Maroun J. Mhanna, MetroHealth Medical Center, 2500 MetroHealth Dr., Cleveland, OH 44109. email: mmhanna@ metrohealth.org. 